\title{
Erratum
}

M. Galehassadi, M. Mahkam, and F. Hosseinzadeh

\section{Erratum to: Synthesis and characterization of new macromolecule systems for colon-specific drug delivery}

https://doi.org/10.1515/epoly-2020-0035

Erratum to: Galehassadi M, Mahkam M, Hosseinzadeh F. Synthesis and characterization of new macromolecule systems for colon-specific drug delivery. e-Polymers. 2007;7(1):28. DOI: 10.1515/epoly.2007.7.1.314.

The university name in the affiliation "Azarbaijan University of Tarbiat Moallem, Tabriz" was misspelled as “Azarrbaijan University of Tarbiat Moallem Tabriz”. The corrected affiliation of authors appears below.

Department of Chemistry, Azarbaijan University of Tarbiat Moallem, Tabriz, Iran 